\title{
Modelado del impacto de bala en una placa de acero 1045
}

\section{Modeling the impact of a bullet on a 1045 steel plate}

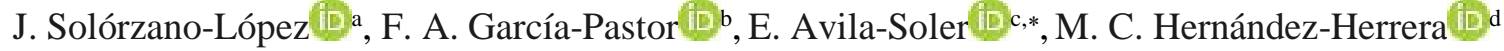 \\ a Departamento de Metalurgia, Universidad Autónoma de México, Ciudad de México, México. \\ ${ }^{b}$ Departamento de Ingeniería Metalúrgica, Cinvestav Saltillo, Coahuila, México. \\ c Departamento de Sistemas, Universidad Autónoma Metropolitana, Ciudad de México, México. \\ d Subdirección de Planeación y Vinculación, Tecnológico Nacional de México-ITGAM, Ciudad de México, México.
}

\begin{abstract}
Resumen
El modelado matemático es una herramienta poderosa, especialmente cuando se analizan fenómenos complejos y potencialmente peligrosos, como impactos de bala. En este artículo, se presentan los resultados de un modelo de elemento finito del impacto de una bala disparada a larga distancia $500 \mathrm{~m}$ y baja velocidad de calibre 0.223 que impacta contra un blanco de acero 1045. Se simulan varios ángulos de impacto, que muestran una penetración de bala y una deformación superior al 50\%. Los impactos del proyectil que rozan el borde de la placa, permiten cuantificar el campo de esfuerzos residuales sobre el objetivo. Estas tensiones residuales son una indicación numérica de la fuerza del impacto.
\end{abstract}

Palabras Clave:

Modelado matemático, bala, impacto, placa de acero.

\begin{abstract}
Mathematical modeling is a powerful tool, especially when analyzing complex and potentially dangerous phenomena, such as bullet impacts. In this paper, the results of a finite element model of the impact of a long-distance $500 \mathrm{~m}$, low speed 0.223 caliber bullet impacting a steel blank are presented. Several impact angles are simulated, showing bullet penetration and deformation larger than $50 \%$. The results show impacts with penetrations or incrustations in the substrate or white plate and that cause deformations greater than 50\%. The impacts of the projectile just touching the edge of the plate, allow to quantify the field of residual efforts on the target. These residual stresses are a numerical indication of the force of the impact.
\end{abstract}

Keywords:

Mathematical modelling, bullet, impact, steel plate.

\section{Introducción}

La balística es una disciplina que se ocupa del análisis del funcionamiento de las armas de fuego y los efectos de sus proyectiles (Kneubuehl, 2011). El presente estudio está dirigido a obtener información que permita analizar los efectos de penetración de los proyectiles en el material blanco ya que de esto es un indicativo del poder de la ojiva, misma que es diseñada para penetrar y destruir; aumentar el alcance de los mismos e incluso analizar el tipo de impactos de las ojivas que ayuden a mejorar los métodos existentes de protección contra ellas.

Existe una gran cantidad de literatura sobre balística en general, análisis físico de la misma, caracterización de los impactos (Kneubuehl, 2011), así como el análisis del tipo de impacto en materiales destinados a la protección tanto de personal como de vehículos (Tan, Lim y Cheong, 2003), involucrando fibras modernas como placas de Kevlar o de metal, principalmente acero, aunque también se usan materiales compuestos e inclusive arreglos combinados de acero y aluminio.

Sin embargo, estas obras sólo descriptivas son pronto superadas por obras que modelan específicamente el impacto de las ojivas en cuanto a la fuerza del impacto como los esfuerzos residuales sobre placas sólidas.

Los modelos físicos y/o matemáticos son herramientas muy importantes para el estudio de situaciones complejas o peligrosas en el ámbito industrial o científico. Si bien el uso de un arma siempre conlleva ciertos riesgos, es difícil construir modelos físicos que reproduzcan su funcionamiento, por lo que estos modelos no son tan útiles en el caso de la balística como son los modelos matemáticos. Existen trabajos que describen los efectos del impacto de una bala de forma teórica (Shim, Tan y Tay, 1995), sin embargo, existe una gran cantidad de trabajo (que incluye tanto

\footnotetext{
*Autor para la correspondencia: eas@azc.uam.mx

Correo electrónico: xaxni2006@yahoo.com.mx (Juan Solórzano-López), garcia@cinvestav.edu.mx (Francisco A. García-Pastor), eas@azc.uam.mx (Enrique Avila-Soler), mchernadez@itmgam.edu.mx ( Mary Carmen Hernández-Herrera) 
artículos como actas y tesis) que realizan cálculos matemáticos para simular un plano y sus efectos en diferentes sustratos (Tham, Tan y Lee, 2008)- (Børvik, et al, 2011)]. Tham, Tan y Lee (2008) informan los resultados de la simulación del impacto de proyectiles disparados con un dispositivo de aire comprimido en cascos de Kevlar. Realizan cálculos utilizando el software AUTODYN y registran imágenes de los impactos con fotografía ultrarrápida. Preece (2004) también realiza cálculos, usando AUTODYN, simulando impactos de rifle calibre 0.338 tanto en acero como en Kevlar, reportando profundidades de penetración de proyectiles y determinando que las simulaciones que involucran Kevlar no tuvieron éxito. La simulación de tejidos conlleva muchos problemas por la naturaleza de su conformación, ya que no son sólidos rígidos sino flexibles e incluso elásticos. Barauskas y Abraitiene (2007) realizan cálculos aplicando un modelo llamado mezzomecánico, que permite analizar un tejido de una forma más cercana a la realidad. También aplican modelos plásticos, elásticos y cinemáticos, obteniendo resultados que coinciden con los obtenidos experimentalmente. Ramavat, Thorat y Prashant (2012) realizan cálculos que simulan impactos de bala en puertas blindadas de acero utilizando un método patentado al que llaman análisis de radio no lineal explícito e informan una buena concordancia entre los resultados de la simulación y las determinaciones experimentales. Børvik, Dey y Clausen (2009) realizaron simulaciones de impacto de proyectiles de calibre 7.62 en láminas de acero, aplicando el elemento finito con los modelos Johnson-Cook y Cockroft-Latham. Chelluru (2007) en su tesis, también aplica Elemento Finito con el código LS DYNA y obtiene buenos resultados en la simulación de impactos balísticos sobre placas de acero. Adams (2006) utiliza Elemento Finito por triplicado, ya que el autor realiza simulaciones con tres códigos: MSC DYTAN, ABAQUS y LS DYNA y dos calibres: 5.56 y 7.62. Los resultados varían entre uno de los códigos, aunque en general hay un buen acuerdo. Børvik, et al (2011) en otro trabajo, realizan simulaciones de impactos de bala en superficies metálicas, pero variando el ángulo de disparo, de $0^{\circ}$ (perpendicular) a $60^{\circ}$. Todos los trabajos anteriores tienen impactos de proyectiles a velocidades que van desde 205 a $830 \mathrm{~m} / \mathrm{s}$, es decir, altas velocidades. Sháněl y Španiel (2014) realizaron un análisis de algunos resultados experimentales del impacto de una bala en una armadura compuesta junto con el modelado numérico de enfoques. Se desarrollaron modelos de una sección de armadura sándwiches (cara de ataque y posterior) compuestos de alta calidad para la protección balística. Clayton (2017) menciona que se han desarrollado métodos para describir la física del impacto y la balística durante varias décadas. Estos incluyen matemática analítica de representaciones, así como simulaciones por computadora. La prueba de impacto plano se estudia a partir de las perspectivas de soluciones analíticas a las ecuaciones de RankineHugoniot, análisis de onda estable y dinámica finita. Pirvu, Ionescu, Deleanu y Badea (2017) realizan un modelo isotérmico macro FEM en el que simuló el impacto de una bala FMJ de 9 mm en un paquete estratificado para restringir las pruebas preliminares para los paquetes estratificados reales hechos de capas de Twaron LFT SB1plus (Teijin), teniendo en cuenta la fricción entre capas y entre viñetas y capas, con el fin de obtener el número de capas que detienen la bala.

Bodepati (2017) hace una serie de simulaciones del impacto de proyectiles $9 \mathrm{~mm}$ sobre textiles reforzados con polímeros. La distancia de impacto varía desde 1 hasta 10 metros y las simulaciones son validadas con experimentos, encontrando las condiciones en las cuales hubo penetración del textil o bien sólo deformación del mismo. Duodu (2017) presenta los resultados de simular el impacto de un proyectil esférico en un compósito de fibra de carbono y epóxico sobre una placa de acero. El software comercial usado fue ABAQUS, y encontraron que el perfil velocidad/tiempo y la absorción energética coinciden con los reportados. Por último, Soydan (2018) realiza la simulación del impacto de un proyectil de $9 \mathrm{~mm}$ sobre un blanco multicapa de fibra de cemento/kevlar/acero. Determinaron la deformación del blindaje, así como los esfuerzos residuales resultado de dicho impacto. El software usado fue AUTODYN 3D.

Más allá de la información técnica obtenida con los trabajos ya mencionados, las simulaciones numéricas pueden tener otras aplicaciones. Desde un punto de vista criminalístico, por ejemplo, los códigos comerciales serían valiosos asistentes para obtener información sobre impactos en la escena del crimen, para determinar detalles difíciles de observar en un principio. El presente trabajo realiza simulaciones del impacto de una bala sobre un sustrato de acero 1045 a baja velocidad (85 m / s) y con un ángulo de impacto variable, buscando emular el impacto de proyectiles calibre 0.223 disparados a una distancia de $500 \mathrm{~m}$ del objetivo. Los resultados de estas simulaciones se presentan no solo como imágenes, sino también con información sobre los esfuerzos residuales provocados por el impacto.

\section{Desarrollo experimental}

Se realizó por medio de los incisos a al e:

a) El modelo matemático realizado simula el impacto de un proyectil de calibre 0.223 sobre una placa de acero 1045 , con un espesor de $5 \mathrm{~cm}$. El ángulo de impacto varió entre $45^{\circ}, 15^{\circ}$ y $5^{\circ}$, con respecto a la horizontal. La geometría del sistema en estudio se muestra en la Figura 1.

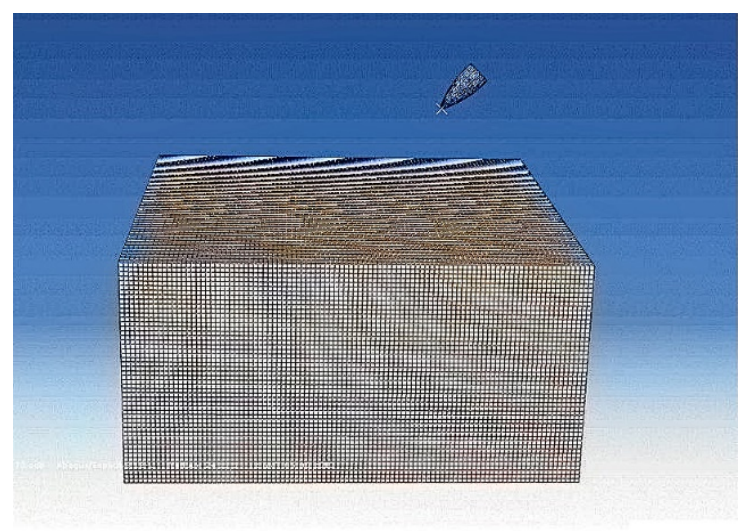

Figura 1: Geometría del sistema en estudio.

b) Las condiciones de modelado son:

I. El objetivo o blanco se considera un cuerpo deformable con propiedades tanto elásticas como plásticas.

II. Los proyectiles se consideran cuerpos rígidos indeformables. Por esta razón, no se simula su respuesta después del impacto.

III. Las simulaciones se realizaron durante el tiempo necesario para lograr la estabilización del material blanco, es decir, cuando los esfuerzos internos del mismo ya no sufran cambios.

c) Las ecuaciones gobernantes que se resolvieron numéricamente fueron: ecuaciones de movimiento (1), incremento de tiempo (2), energía interna como función de la temperatura (3), modelo de JohnsonCook y sus parámetros (4) y la presión hidrodinámica desarrollada durante el impacto (5). 
Esta resolución se llevó a cabo con el método explícito de integración de diferencias centrales. En todas las ecuaciones, v representa la velocidad, a representa la aceleración mientras que t representa el tiempo. El subíndice i determina los pasos del incremento de tiempo.

$$
\begin{aligned}
& v^{(i+1 / 2)}=v^{(i-1 / 2)}+\frac{\Delta t^{(i+1)}+\Delta t^{(i)}}{2} a \\
& s^{(i+1)}=s^{(i)}+\Delta t^{(i+1)} v^{(i+1 / 2)} \\
& \Delta t \leq \frac{2}{\omega_{\max }} \\
& E=\frac{1}{V_{0}} \int C_{V} d T \approx \frac{C_{V}\left(T-T_{0}\right)}{V_{0}} \\
& \sigma_{y}=\left(\varepsilon_{p}, \dot{\varepsilon}, T\right)=\sigma_{0}\left[1+\frac{B}{\sigma_{0}}\left(\varepsilon_{p}\right)^{n}\right]\left[1+C \ln \left(\dot{\varepsilon}^{*}\right)\right]\left[1-\left(T^{*}\right)^{m}\right] \\
& \dot{\varepsilon}^{*}=\frac{\dot{\varepsilon}}{\dot{\varepsilon_{0}}} T^{*}=\frac{\left(T-T_{r}\right)}{\left(T_{m}-T\right)_{r}} \quad \sigma_{0}=\exp \left(A_{1} R_{C}+A_{2}\right) \\
& p=\frac{\rho_{0} C_{0}^{2}(\eta-1)\left[\eta-\frac{\Gamma_{0}}{2}(\eta-1)\right]}{\left[\eta-S_{a}(\eta-1)\right]^{2}}+\Gamma_{0} E \quad \eta=\frac{\rho}{\rho_{0}}
\end{aligned}
$$

Se describen el significado de las notaciones de las ecuaciones en la tabla 1.

Tabla 1: Notaciones de las ecuaciones

\begin{tabular}{ll}
\hline \multicolumn{1}{c}{ Notación: } & \multicolumn{1}{c}{ Significado } \\
\hline$\Gamma_{\mathrm{O}}$ & $\begin{array}{l}\text { Gamma de Gruneisen en el estado de } \\
\text { referencia }\end{array}$ \\
$\dot{\varepsilon_{\mathrm{O}}}$ & Tasa de deformación de referencia \\
$\varepsilon_{\mathrm{P}}$ & Deformación plástica equivalente \\
$\dot{\varepsilon}$ & Tasa de deformación plástica \\
$\varepsilon^{*}$ & Tasa de deformación adimensional \\
$\mathrm{H}$ & Densidad adimensional \\
$\mathrm{P}$ & Densidad \\
$\rho_{\mathrm{O}}$ & Densidad inicial \\
$\sigma_{\mathrm{y}}$ & Término del modelo de Johnson-Cook \\
$\omega_{\mathrm{max}}$ & Valor propio máximo del sistema \\
$\mathrm{A}_{1}, \mathrm{~A}_{2}, \mathrm{~B}, \mathrm{C}$, & Parámetros de material \\
$\mathrm{n}_{\mathrm{m}, \sigma_{\mathrm{O}}}$ & Aceleración \\
$\mathrm{A}$ & Velocidad del sonido en el material \\
$\mathrm{C}_{\mathrm{O}}$ & Calor específico a volumen constante \\
$\mathrm{C}_{\mathrm{V}}$ & Energía interna \\
$\mathrm{E}$ & Dureza Rockwell C del material \\
$\mathrm{R}_{\mathrm{C}}$ & Coeficiente de pendiente de Hugoniot lineal \\
$\mathrm{S}_{\alpha}$ & Términos de la ecuación de movimiento \\
$\mathrm{s}, \mathrm{V}$ & Tiempo \\
$\mathrm{T}$ & Temperatura \\
$\mathrm{T}$ & Temperatura de referencia \\
$\mathrm{T}_{\mathrm{r}}$ & Temperatura de fusión \\
$\mathrm{T}_{\mathrm{m}}$ & Temperatura adimensional \\
$\mathrm{T}^{*}$ & Volumen específico de referencia \\
$\mathrm{V}_{\mathrm{O}}$ &
\end{tabular}

Es importante mencionar que el algoritmo de resolución del modelo matemático es explícito, por lo tanto, la magnitud del paso del tiempo es limitada. En las ecuaciones de movimiento y la magnitud del aumento de tiempo el Omega representa el valor máximo del sistema.

d) Las condiciones de borde. El objetivo es una placa de 10x10x5 cm que está fija y los proyectiles que son cuerpos rígidos que se mueven a $85 \mathrm{~m} / \mathrm{s}$, con ángulos de impacto de $5^{\circ}, 15^{\circ}$ y $45^{\circ}$ con respecto a la horizontal. Los ángulos se determinaron de esa forma para determinar el efecto del impacto con trayectoria no cercana a la trayectoria normal o perpendicular, trayectoria de la que ya hay bastante información en la literatura especializada.

e) Descripción del modelo. Fue desarrollado utilizando el código comercial ABAQUS versión 6.12-1, de forma explícita y dinámica. El objetivo es una placa con dimensiones ya definidas y una malla de 500,000 nodos. El material objetivo es acero 1045 con propiedades mecánicas de $200 \times 10^{9} \mathrm{~Pa}$ del Módulo de Young y densidad de $7860 \mathrm{Kg} / \mathrm{m}^{3}$ (Chelluru). La simulación se realizó durante 0.01 segundos.

\section{Resultados y Discusión}

Una vez realizados los cálculos con las condiciones ya mencionadas, se tienen diversos resultados posteriores al impacto. En la Figura 2 se puede ver que el proyectil penetra completamente en la placa blanca. Si bien es factible calcular las tensiones residuales utilizando el software antes mencionado, en este caso la deformación es superior al 50\%, es decir, siempre se pone el superior al $50 \%$ como un parteaguas de que no hay ambigüedad de ningún tipo, porque no se está diciendo nada como "superior al 50\%" ya que se refiere a la zona del impacto.

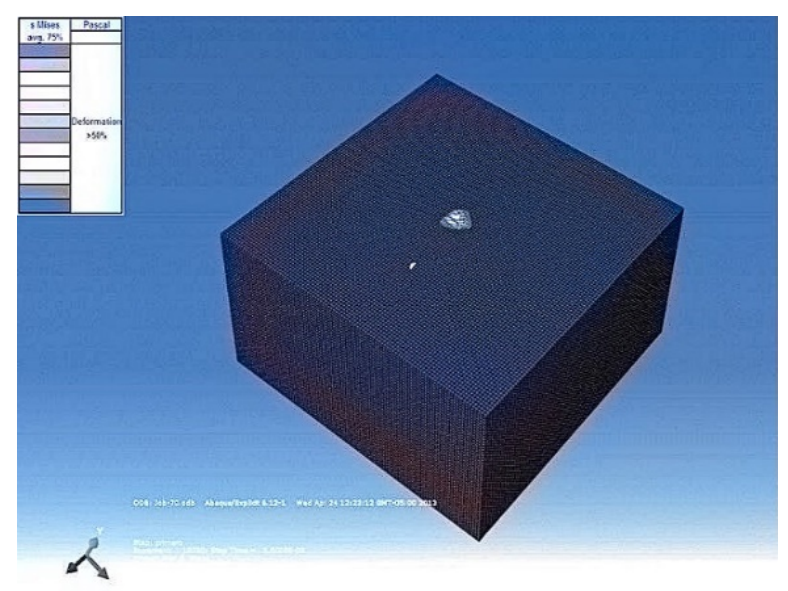

Figura 2: Impacto a $45^{\circ}$. El proyectil está completamente alojado en el objetivo.

En la figura 3 se muestra el gráfico de tensiones residuales con respecto a la posición, las medidas son en relación al inicio de la influencia del impacto a $45^{\circ}$ y corresponde a la dirección del impacto. En este gráfico se aprecia que no existe simetría en los esfuerzos, debido al ángulo de impacto. 


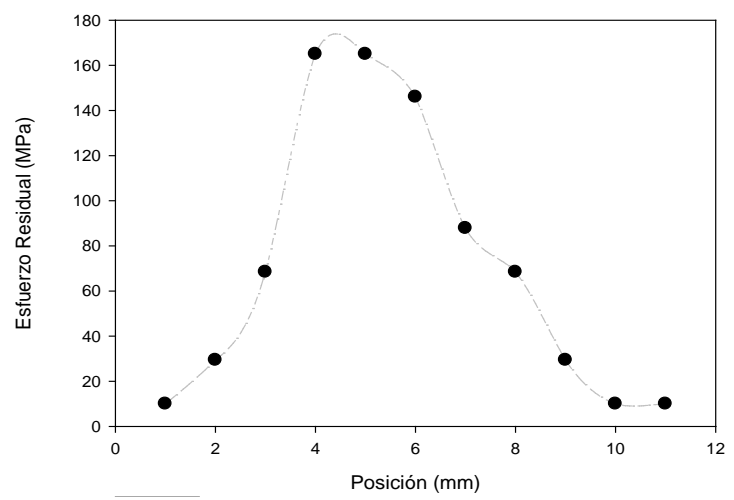

Figura 3. Impacto a $45^{\circ}$. Grafica del esfuerzo residual contra la posición.

La figura 4 muestra el resultado del impacto a $15^{\circ}$, siempre con respecto a la horizontal y a una velocidad de contacto de 85 $\mathrm{m} / \mathrm{s}$. La deformación de la placa blanca se observa por la fuerza del impacto. Como en el cálculo anterior, la deformación es superior al $50 \%$, es decir, siempre se pone el superior al $50 \%$ como un parteaguas de que no hay ambigüedad de ningún tipo, porque no se está diciendo nada como "superior al 50\%" ya que se refiere a la zona del impacto.

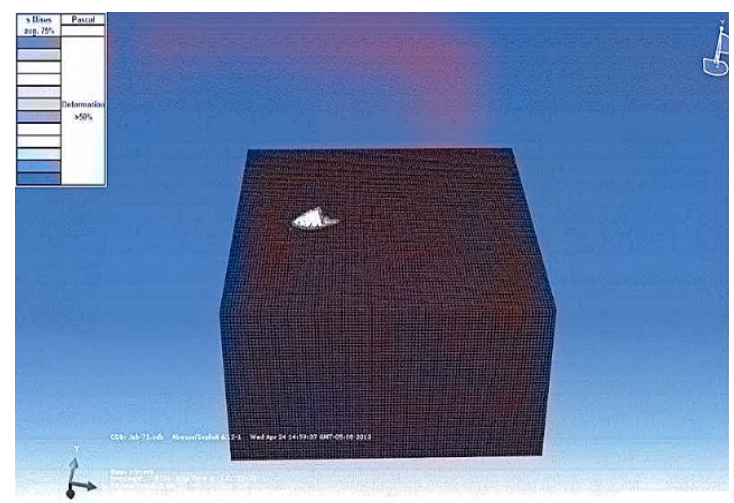

Figura 4: Impacto a $15^{\circ}$. El proyectil está incrustado superficialmente en el objetivo.

La Figura 5 muestra el gráfico de tensión residual según la posición, las medidas son en relación al inicio de la influencia del impacto y corresponde a la dirección del impacto. En este gráfico se aprecia que no existe simetría en los esfuerzos, debido al ángulo de impacto.

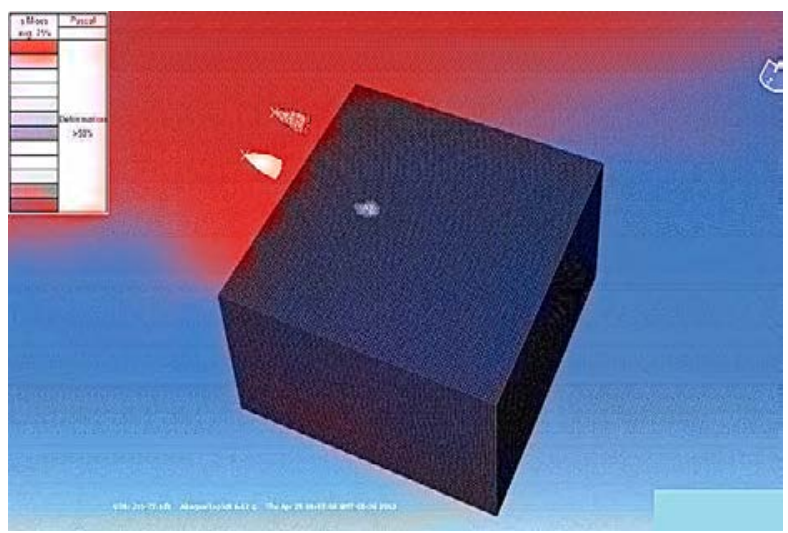

Figura 5: Impacto a $5^{\circ}$. El proyectil rebota en la superficie del objetivo.
La figura 6 muestra el gráfico de tensiones residuales del impacto correspondiente a $5^{\circ}$ con respecto a la horizontal. En este gráfico es notable la simetría de la distribución de tensiones residuales, que a pesar del ángulo de impacto tiene un rebote debido a la geometría del proyectil.

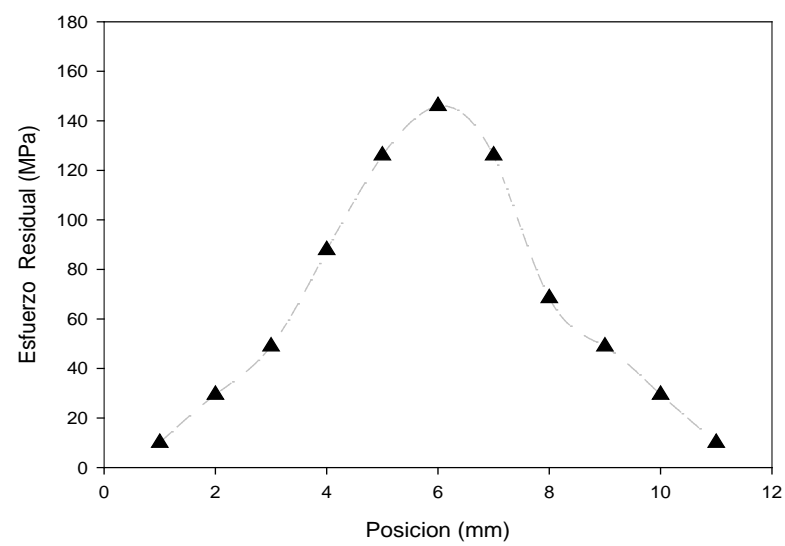

Figura 6: Impacto a $5^{\circ}$. Grafica de esfuerzo residual contra la posición.

En la siguiente figura (figura 7), se muestra el resultado de un cálculo realizado con un impacto muy leve contra la placa blanca de tal manera que se pudieron registrar las tensiones residuales. El proyectil pasa tocando el borde de la placa (el contacto entre uno no supera el milímetro de longitud) y la deformación es despreciable. El rango de tensiones residuales registradas varía de $2.24 \times 10^{8}$ a $1.00 \times 10^{7}$ Pascales.

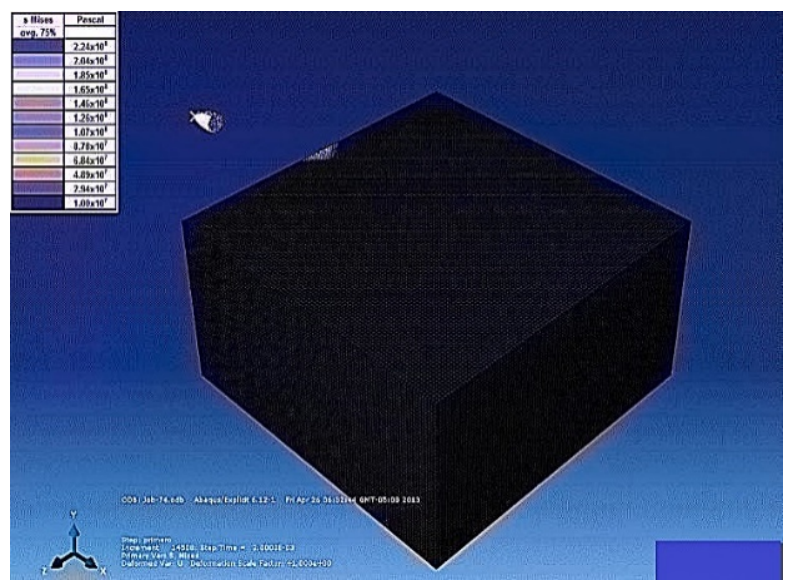

Figura 7: Impacto a $5^{\circ}$ y en el borde del objetivo. Se aprecia la huella de tensiones residuales que deja la fricción del proyectil.

La Figura 8 muestra el gráfico de tensiones residuales con respecto a la posición del impacto del proyectil a $5^{\circ}$ en el borde del objetivo. En este gráfico se visualiza la similitud con el gráfico 6, donde el aumento de esfuerzos desde el inicio de la pista es muy similar; mientras que la disminución está limitada por la proximidad al borde que provoca un deterioro considerable. 


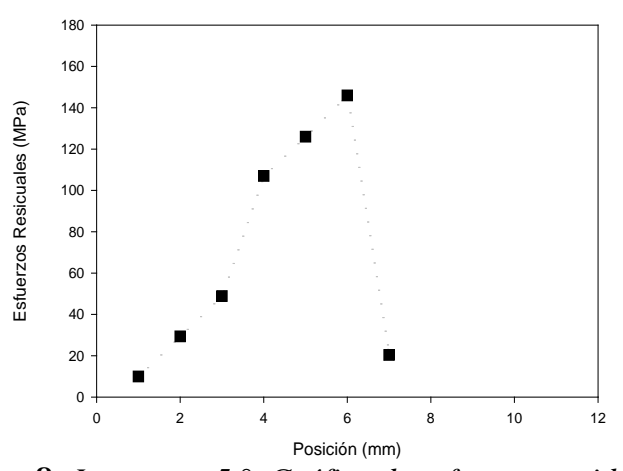

Figura 8: Impacto a $5^{\circ}$. Gráfica de esfuerzos residuales contra posición.

La figura 9 muestra el resultado de un cálculo similar al de la figura 5, con la salvedad de que en este caso se trata de un doble impacto con una longitud de $3 \mathrm{~mm}$ de contacto entre el borde de la placa y el proyectil. Sin embargo, esta longitud de contacto es suficiente para provocar deformaciones superiores al 50\% en el objetivo y, por tanto, no es factible registrar los valores de tensión residual.

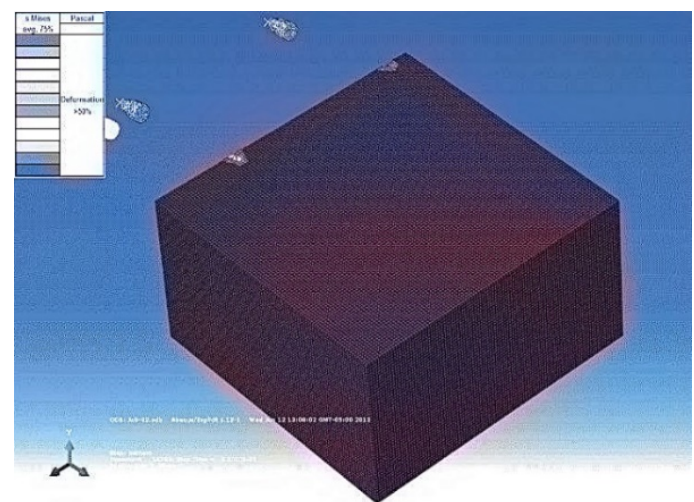

Figura 9: Doble impacto a $5^{\circ}$ y en el borde del objetivo. Se aprecian rastros de tensiones residuales dejadas por la fricción del proyectil.

Los resultados obtenidos permiten determinar información relativa a impacto a larga distancia (baja velocidad) de ojivas calibre 0.223 sobre un blanco de acero 1045. En la literatura no hay datos a este respecto y menos aún en lo referente a los ángulos de impacto seleccionados, debido principalmente a que investigaciones previas se han enfocado a impactos con ángulos muy cercanos a la perpendicularidad (trayectoria normal cercana o igual a $90^{\circ}$ ). Las gráficas de esfuerzos residuales permiten determinar que la simetría de los mismos con respecto a la longitud está en función del ángulo de impacto. Esto se puede explicar en función de la incidencia de la ojiva, a $45^{\circ}$ la bala se incrusta en el blanco y hay mucha energía que actúa en la zona frente a la punta de ésta. Cuando la bala roza simplemente la orilla, el rozamiento ocurre debido al contacto de la base de la ojiva con la orilla de la placa blanco, por lo que la deformación y los esfuerzos residuales resultantes existen en la zona posterior al impacto. Por último, también se puede explicar la simetría de los esfuerzos residuales en función de un impacto superficial o rebote de la ojiva en la zona central del blanco. En este caso particular, se tiene que la geometría del impacto es simétrica no solo en la huella de deformación sino en la misma distribución de esfuerzos. Ésta información (cuantificada por medio del código ABAQUS) no ha sido encontrada en la literatura, abocada en experimentar otro tipo de impactos, diferentes en la naturaleza material del blanco como en el ángulo de impacto de la ojiva. Así mismo, la baja velocidad que emula un disparo a larga distancia determina el comportamiento de la ojiva y del blanco en condiciones no usadas en otros experimentos y reportes científicos e ingenieriles.

\section{Conclusiones}

El modelo matemático realizado en este trabajo muestra los resultados de impactos de proyectiles calibre 0.223 disparados a 500 metros de distancia. Estos proyectiles provocaron deformaciones en la placa blanca de magnitud superior al 50\%, en todos los casos excepto 1 , se registran valores de tensión residual entre $2.24 \times 10^{8}$ y $1.00 \times 10^{7}$ Pascales.

Respecto a las gráficas de esfuerzo residual contra posición, se puede decir que existe simetría en el impacto singular a ojiva no se incrusta y con ángulo de 5 grados, mientras que ésta no es tan perfecta en el mismo ángulo tratándose de impactos dobles a $5^{\circ} \mathrm{o}$ bien en el caso de impactos a $45^{\circ}$, sobre todo por la dirección del impacto por ojiva que queda incrustada. Es decir, la zona donde se sitúa la punta de la ojiva concentra mayor esfuerzo residual.

La mejor simetría corresponde a un impacto a $5^{\circ}$, siempre con respecto a la horizontal, y haciendo blanco a la mitad de la placa de acero 1045. La bala no se incrustó en dicha placa y el impacto describe una simetría muy buena, cuantificada por los esfuerzos residuales calculados.

\section{Conflicto de intereses}

Los autores declaran que no existen conflictos de intereses.

\section{Referencias}

Adams, B. (2006). "Simulation of ballistic impacts on armored civil vehicles". Master Thesis. Eindhoven University of Technology. doi:http://www.mate.tue.nl/mate/pdfs/6290.pdf

Barauskas, R., \& Abraitiene, A. (2007). Computational analysis of impact of a bullet against the multilayer fabrics in LS-DYNA. International journal of impact engineering, 34(7), 1286-1305. doi:https://doi.org/10.1016/j.ijimpeng.2006.06.002

Bodepati, V. M. (2017). Numerical simulation and experimental validation of E-glass/epoxy composite material under ballistic impacto f $9 \mathrm{~mm}$ soft projectile. Procedia $\quad$ engineering(173), doi:http://dx.doi.org/10.1016/j.proeng.2016.12.068

Børvik, T., Dey, S., \& Clausen, A. H. (2009). Perforation resistance of five different high-strength steel plates subjected to small-arms projectiles. International Journal of Impact Engineering, 36(7), 948-964. doi:https://doi.org/10.1016/j.ijimpeng.2008.12.003

Børvik, T., Olovsson, L., Dey, S., Langseth, \& M. (2011). Normal and oblique impact of small arms bullets on AA6082-T4 aluminium protective plates. International Journal of Impact Engineering, 38(7), 577-589. doi:http://dx.doi.org/10.1016/j.ijimpeng.2011.02.001

Chelluru, S. K. (2007). Finite Element Simulations of Ballistic Impact on Metal and Composite Plates. Master Thesis, 1(1), 202. Retrieved 04 11, 2021, from http://hdl.handle.net/10057/1533

Clayton, J. D. (2017). Methods for Analysis and Simulation of Ballistic Impact. Recent Patents on Engineering, 11(1), 49-61. doi:https://doi.org/10.2174/1872212110666161028160205

Duodu, E. G. (2018). Comparison of ballistic impact behavior of carbon fiber/epoxy composite and Steel metal structures. Iranian journal of science and technology, 13-22. doi:https://doi.org/10.1007/s40997-0170072-6

Kneubuehl, B. P. (2011). Wound Ballistics Basics and Applications (Vol. 1). Springer-Verlag Berlin Heidelberg. doi:10.1007/978-3-642-20356-5

Pirvu, C., Ionescu, T. F., Deleanu, L., \& Badea, S. (2017). Simplified simulation of impact bullet - stratified pack for restraining ballistic tests. $\begin{array}{llll}\text { MATEC Web } & \text { Conf. } & \end{array}$ doi:http://dx.doi.org/10.1051/matecconf/201711206023

Preece, D. S. (2004). "Bullet Impact on Steel and Kevlar®/Steel Armor: Computer Modeling and Experimental Data.". Proceedings of the ASME/JSME 2004 Pressure Vessels and PiProblems Involving Thermal Hydraulics, Liquid Sloshing, and Extreme Loads on Structures., 207-212. doi:https://doi.org/10.1115/PVP2004-3050 
Ramavat, S., Thorat, D., \& Prashant, C. (2012). "Simulation of bullet impact on bullet resistant steel plate". (G. \&. Co.Ltd, Ed.) Simulation Driven Innovation, HTC 2012, 1-13. Retrieved 04 11, 2021, from http://dspace.nitrkl.ac.in:8080/dspace/bitstream/2080/3244/1/2019_ICO RT_DPJena_Simulation\%20of\%20Bullet.pdf

Sháněl, V., \& Španiel, M. (2014). Ballistic Impact Experiments and Modelling of Sandwich Armor for Numerical Simulations. Procedia $\begin{array}{lll}\text { Engineering, } & 79, & 230-237 .\end{array}$ doi:https://doi.org/10.1016/j.proeng.2014.06.336

Shim, V. P., Tan, V. B., \& Tay, T. E. (1995). Modelling deformation and damage characteristics of woven fabric under small projectile impact. International Journal of Impact Engineering, 16(4), 585-605. doi:https://doi.org/10.1016/0734-743X(94)00063-3
Soydan, A. T. (2018). Simulation and Experimental Tests of Ballistic Impact on Composite Laminate Armor. Advances in materials science and engineering, 2018, 12. doi:https://doi.org/10.1155/2018/4696143

Tan, V. B., Lim, C. T., \& Cheong, C. (2003). Perforation of high-strength fabric by projectiles of different geometry. International Journal of Impact Engineering, 28(2), 207-222. doi:https://doi.org/10.1016/S0734743X(02)00055-6

Tham, C. Y., Tan, V., \& Lee, H. P. (2008). Ballistic impact of a KEVLAR ${ }^{\circledR}$ helmet: Experiment and simulations. International Journal of Impact Engineering, 35(5), doi:https://doi.org/10.1016/j.ijimpeng.2007.03.008
304-318. 